\title{
Use of a text messaging service for communication with parents and carers
}

\author{
Caroline Palmer
}

\section{Citation}

Palmer C (2019) Use of a text messaging service for communication with parents and carers. Primary Health Care. doi: 10.7748/phc.2019.e1472

\section{Peer review}

This article has been subject to external double-blind peer review and has been checked for plagiarism using automated software

\section{Correspondence caroline.palmer@leicspart. nhs.uk}

\section{Conflict of interest}

None declared

\section{Accepted}

10 September 2018

Published online March 2019

\section{Permission}

To reuse this article or for information about reprints and permissions, please contact permissions@rcni.com

\begin{abstract}
This article looks at how parents and carers can use text messaging to have safe and secure conversations with health visitors about a range of issues. It describes how a new text messaging enquiries service for parents and carers was successfully adopted by staff and service users, achieving a high level of uptake over a short period of time.

The article also discusses how a text messaging service of this nature can extend a service's reach, offer support that may not have been previously sought, help users to make informed and objective health choices, and support staff to work more efficiently, while offering users greater flexibility in how they interact with health professionals.

The service changes described in this article took place at Leicestershire Partnership NHS Trust, which set up a text messaging enquiries service in November 2016 to support parents or carers of babies and children aged 0-5 years, in readiness for launching its new Healthy Together 0-19s team.
\end{abstract}

\section{Author details}

Caroline Palmer, ChatHealth clinical lead, Leicestershire Partnership NHS Trust, Leicester, England

\section{Keywords}

communication, information technology, health visitors, patient engagement, patient education, patient choice, child health, school nurses, service redesign, service improvement, parents, carers

\section{Introduction}

We live in an increasingly digital world. People use technology and digital platforms every day to manage their lives, personally and professionally. Ofcom (2017) recognised that: 'In today's connected world, people increasingly expect to be able to access online services whenever they want and wherever they are.' Furthermore, Ofcom (2017) highlighted that $94 \%$ of adults own or use a mobile phone.

Healthcare has historically been provided predominantly face-to-face. This helped practitioners to provide high-quality care. However, in today's digital world, service users demand greater choice and ease of use. The National Information Board (2014) highlighted that: 'The use of digital technologies across all social groups under the age of 60 demonstrates that there is a latent demand to use these technologies to improve management of health and access to care services.'

NHS England (2017a) showed that in the past ten years, people's expectations of how they can access services have changed. It stated that being able to access a service online or by telephone should be a minimum standard, and encouraged greater moves towards this in the near future, providing changes do not dilute the importance of face-to-face care: 'Over the next two years the NHS will make very significant steps towards increasing how its services can be accessed online, while remembering that healthcare is about people and that many patients want and need the reassurance of a real person to talk to face to face.'

NHS England (2017b) also recognised that: 'Digital technology can accelerate more sophisticated and acceptable service delivery, data capture, interpretation and support, and have a very significant transformational and long-term impact.'

\section{A new digital tool}

Recognising that it needed to respond to changes in its service users' behaviour and attitudes towards access, Leicestershire Partnership NHS Trust developed ChatHealth, a secure, web-based tool for receiving and responding to text messages from mobile phones (Endicott and Clarke 2014). The trust initially examined off-the-shelf products to see 
if it could safely and securely address the needs of messaging in healthcare. However, it could not find one that fulfilled all its requirements, so developed ChatHealth itself.

When developing the tool, the trust consulted the police, the Royal College of Nursing (RCN), health professionals, and service users to ensure it was secure and fit for purpose. It was designed so that health professionals responding to service-user messages could only connect to ChatHealth's online management portal using the NHS's secure network. The National Institute for Health and Care Excellence reviewed ChatHealth, while NHS Digital assessed it technically and the trust assessed it for compliance with the NHS's then digital clinical risk management standard, SCCI0129 (now DCB0129).

After being successfully used for several years by the school nurses for young people aged 11-19 years in Leicester, Leicestershire and Rutland, the opportunity arose for the service to be adopted by the whole of the 0-19 service in this area, including health visitors for parents of newborns and under fives.

To evaluate service users' needs, the trust consulted parents and carers using focus groups in community venues. They reported that they sometimes struggled to get hold of health visitors and often did not want to bother them with simple questions to which they felt they should already know the answers.

Parents and carers said they wanted quick and easy access to their health visitor, with some stating that they had busy working lives, which sometimes made it difficult to access services. Some parents also said they sometimes felt less comfortable accessing healthcare faceto-face, as asking questions embarrassed them.

It was therefore important to offer them choice in how they could access services, thoroughly evaluate changes to service delivery, and regularly obtain feedback from staff and service users.

\section{A pilot text messaging enquiries service}

The trust decided to respond to parents and carers' needs by using ChatHealth to provide them with a text messaging enquiries service. It first piloted the service in five areas that were spread across geographically, demographically and ethnically diverse county and city sites in Leicester, Leicestershire and Rutland. Supported by a school nurse, who was already experienced in running a successful ChatHealth service for young people aged 11-19 years, the health visiting pilot service was initially launched by two health visitors. They received training to ensure they felt comfortable and confident using ChatHealth to respond to messages. The health visitors continued to follow a robustly governed process that took into account clinical and information governance for both staff and service user safety.

Promotional materials were distributed throughout the pilot areas, including business cards, stickers for children's personal health records (red books), posters and pens. This material informed parents and carers of the number to which they could send their enquiries and that they could expect a response from a health visitor within 24 hours during the working week. However, the response time was usually quicker than this.

To use the service, parents and carers sent text messages from their mobile phones to the service's dedicated text number. ChatHealth automatically replied to messages it received, acknowledging receipt, outlining likely response times and providing the terms of service. The automatic replies also directed users to alternative sources of help, if they required it.

ChatHealth provides shared inboxes, which helps teams to share responsibility for conversations and ensure no messages are missed. A duty system was used to manage all incoming messages, which was efficient and time-effective as it only needed two health visitors to run it per day.

The duty health visitors received alerts by either text or email when new messages arrived. They then responded to the messages using ChatHealth. Staff were equipped with a standard operating procedure that provided rules to follow, explained safe approaches to using messaging with service users, and detailed what to do when messages involved heightened safeguarding implications or increased risk.

Parents and carers could ask questions anonymously, if they wanted. Initially, the health visitors felt a little uncomfortable with this, but soon likened it to a casual contact in which they gave quick advice, but did not know the identity of the parent, carer or child. Health visitors recognised they already had similarly anonymous contacts at health promotion events, for example.

All conversations were stored and documented in line with national and local data-protection and record-keeping guidelines, whether the contact was a named user or anonymous.

\section{Key points}

- Digital platforms can offer quick and easy access to healthcare

- Increased contact from parents and carers who may find it difficult to seek support and advice

- Extends service provision to parents and carers who are harder to reach

- Efficient and effective way for health visitors to communicate with parents and carers 


\section{Results}

Between the start of the pilot for parents and carers in November 2016 and December 2017 when the service was using ChatHealth in everyday practice, the team were able to provide the parents and carers of around 140,000 babies and children with access to support. The service received 1,448 enquiries and handled 5,124 messages.

Health visitors responded to a wide range of issues raised by parents and carers (Table 1). These included questions and concerns about breastfeeding, general health, constipation, immunisations, sleep and feeding. Several fathers contacted the service, which is positive as contact has been limited historically with fathers. Health visitors requested feedback from parents and carers after every contact by text message. Almost all feedback from the 460 respondents was very positive, with $97 \%$ stating that they were satisfied or very satisfied with the care provided.

The service ran Monday to Friday, 9am-5pm. Parents and carers reported that they were happy as they could text at any time and their enquiry would get picked up within 24 hours during the working week.

\section{Table 1. Messaging contacts by type}

\begin{tabular}{|l|c|}
\hline Messaging contacts by type & $\begin{array}{c}\text { Percentage } \\
\text { of messages }\end{array}$ \\
\hline General health & $27 \%$ \\
\hline Administration & $14 \%$ \\
\hline Formula feeding & $13 \%$ \\
\hline Constipation/stool & $10 \%$ \\
\hline Breastfeeding & $9 \%$ \\
\hline Parenting advice or support & $9 \%$ \\
\hline Sleep & $6 \%$ \\
\hline Dietary/nutritional/health/lifestyle & $4 \%$ \\
\hline Vitamins & $2 \%$ \\
\hline Immunisations & $2 \%$ \\
\hline Emotional health and well-being & $2 \%$ \\
\hline Signposting & $0.60 \%$ \\
\hline Smoking cessation & $0.60 \%$ \\
\hline Child development & $0.50 \%$ \\
\hline Sexual health & $0.30 \%$ \\
\hline
\end{tabular}

The feedback also highlighted that:

" Messaging is quick and provides easy access for parents and carers to health visitors.

॥ Service users were more engaged, asking questions they may not have previously asked.

॥ The service reassured parents.

\section{Parent case study}

\section{What made you contact the} messaging service?

I used the messaging service because I wanted advice about my 20-month-old daughter.

I was struggling with her being fussy with her eating and so found that I was giving her snacks to eat throughout the day, just so I knew she had eaten something. She was also not sleeping very well at night. I was feeling tired and frustrated and needed to talk to someone.

I had not seen my health visitor since my daughter's one-year check and I did not know whether they would think I should have done something different so I felt nervous to call them or see them face-to-face. But I saw the messaging service number in my GP reception.

\section{Tell us a bit about what happened}

I sent a text message to the messaging service asking for some advice and support and got a quick and friendly response from a health visitor - I was able to ask without feeling like I was pestering them and had the advice I needed really promptly.

\section{What would your feedback be about} the service?

I was able to ask questions comfortably that I felt I may be judged for asking face-toface as I thought I should already know the answers as a mum.

\section{Health visitor case study}

The issue the parent/carer was experiencing and seeking advice for A mother messaged, asking for advice with breastfeeding. She was feeling unsure about whether she was feeding correctly and appeared to lack confidence in her ability.

The care provided by the health visitors staffing the messaging service

The mother was offered support, practical advice and guidance. Her feelings around breastfeeding were discussed and it became apparent that she was feeling low in mood. I was able to gain permission to contact her health visitor and arrange a home visit for further mental health support.

\section{The outcomes of the care provided to} the parent/carer

The mother's immediate concerns regarding breastfeeding were addressed and a plan put in place to offer face-to-face support for low mood from her named health visitor. 
Example comments include:

'I love this service. It is great to be able to text as I can ask questions I am not comfortable raising on the phone or in person, especially little problems that I would not normally ask. It just gives me peace of mind' (Participant 1).

'I am a million per cent satisfied, thank you, 5/5. You did more than enough, thank you' (Participant 2).

'Quick response, easy to use and efficient. I would use this service again and recommend to others that would find it useful. Thank you' (Participant 3).

\section{Service expansion}

Following the success of the pilot text messaging service for parents and carers in the five areas within Leicester, Leicestershire and Rutland, the ChatHealth service has grown.

Health visitors in the other areas from the organisation recognised that the service fulfilled particular needs and provided benefits to staff, parents and carers. They approached the staff users involved in the pilot service to see if it could be expanded to their areas.

The service is now available across all the trust's localities and receives 10-14 new messages each day. Across the board, health visitors have embraced the new way of working and have undertaken the appropriate training and promoted the service.

ChatHealth surveyed 183 staff who used the service to provide advice and support to service users, to ask them what they thought were its main benefits. The feedback received reported that it:

» Is quick and easy.

॥ Improves job satisfaction because it has an immediate effect.

॥ Is time-efficient.

॥ Is safe and they feel supported in using it.

\section{Conclusion}

The aim of offering a text messaging service such as this is not to replace face-to-face care, but to provide a tool that professionals can offer service users to choose how they access healthcare support and advice.

Using text messaging with service users can be a daunting prospect for some health visitors, because it is commonly a new way of working for them and different from how they have previously provided care. However, the NHS encourages us to respond to the ever-changing world in which we deliver care and this can sometimes push us outside our comfort zones.

One staff member commented: 'I was initially anxious about using the messaging service. I have been a health visitor for over ten years and always delivered care in predominantly a face-to-face way. However, I now love the messaging service and enjoy being one of the duty nurses and cannot recommend the service enough.'

The Leicestershire Partnership NHS Trust pilot demonstrated that given the choice, parents and carers will use a text messaging service to seek health advice and support from health visitors. Similarly, given a choice, staff may willingly participate in such an improvement to service.

\section{References}

Endicott J, Clarke M (2014) Nurses in step with the smartphone generation. Primary Health Care. $24,8,20-24$.

National Information Board (2014) Personalised Health and Care 2020. A Framework for Action. www.gov.uk/government/publications/ personalised-health-and-care-2020 (Last accessed: 7 March 2019.)

NHS England (2017a) Next Steps on the NHS Five Year Forward View. www.england.nhs.uk/ publication/next-steps-on-the-nhs-five-yearforward-view (Last accessed: 7 March 2019.)
NHS England (2017b) Five Year Forward View for Mental Health - One Year On Report. www. england.nhs.uk/publication/five-year-forwardview-for-mental-health-one-year-on-report (Last accessed: 7 March 2019.)
Ofcom (2017) The Communications Market Report 2017. www.ofcom.org.uk/research-anddata/multi-sector-research/cmr/cmr-2017 (Last accessed: 7 March 2019.) 